\title{
Initiation in gigacycle fatigue related to metallurgical defects
}

\author{
C. Bathias, A.Nikitin, I.Marines, Q.Wang, C. Wang, \\ University of Paris/ LEME and Sichuan University, China
}

Abstract. This paper is dealing with the fish eye formation around defects in gigacycle regime: mechanisms, modelling, phase transformation.

\section{Introduction}

When the fatigue life is between $10^{6}$ and $10^{7}$ cycles the plastic deformation depends on the plane stress surface effect which explains the critical location of fatigue initiation Typically the initiation starts with one crack only, from the surface. However approaching $10^{9}$ cycles the plastic deformation in plane stress conditions is vanishing; the macroscopic behaviour of the metal is elastic except around metallurgical defects. In very high cycle fatigue, the plane stress conditions are not enough for a surface plastic deformation. The initiation may be located in an internal zone. When the crack initiation site is in the interior this leads to the formation of a fish eye on the fracture surface, typical of gigacycle fatigue. Since the probability of occurrence of a flaw is greater within an internal volume than at a surface, the typical initiation in Gigacycle fatigue will be most often in the bulk in the metal.

\section{Modelling fish eye}

When the crack initiation site is in the interior, this leads to the formation of a "fish-eye" on the fracture surface, and the origin of the fatigue crack is an inclusion, a "super grain" (micro structural homogeneity), or porosity. In almost all cases, this fish-eye appears circular, with a dark area in the centre . Some authors have named: "Optically Dark Area (ODA)" by Y.Murakami, "Fine Granular Area (FGA)" by T. Sakai, "Granular Bright Facet (GBF) by Shiozawa [1].

The fractographic observations show several zones ( figure 1) to support a mechanical model:

- a dark area zone (so-called ODA)due to the initiation mechanisms. [2]

- a penny-shaped zone (short crack growth). Whatever the crack initiation site (spherical or elongated inclusion, super-grain, pore), the fracture surface becomes circular around the initiation site.

- A zone with small radial ridges corresponding to the short to long crack transition

- A zone with large radial ridges (long crack growth). In this zone, the fatigue crack propagation produces striations for which the mean distance between striations is a function of $\Delta \mathrm{K}^{2}$, in good agreement with the CTOD. [2]

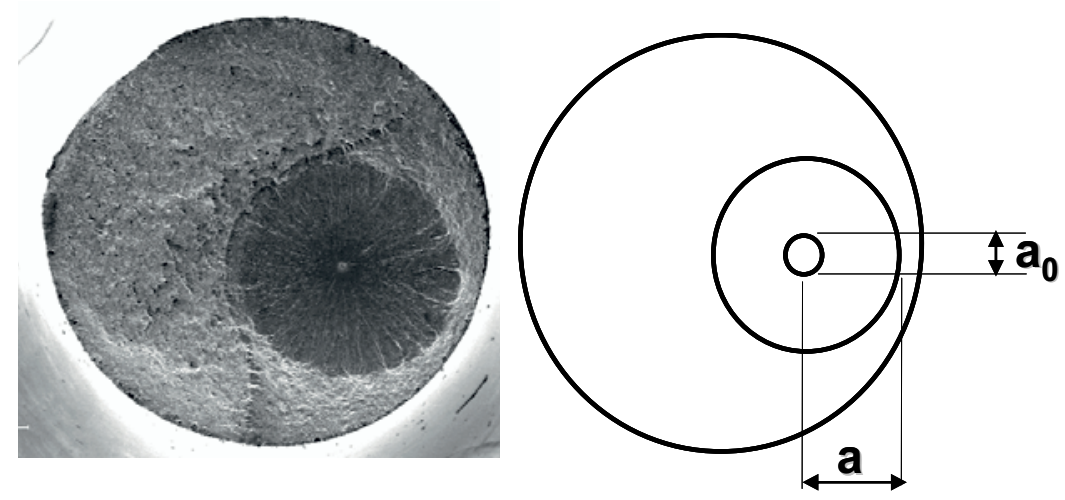

Figure 1. Typical fish eye with initiation and short crack growth

Corresponding author: claude@bathias.com

This is an Open Access article distributed under the terms of the Creative Commons Attribution License 4.0, which permits unrestricted use, distribution, and reproduction in any medium, provided the original work is properly cited. 
The integration of the Paris Hertzberg law without transition, to determine the crack growth life will begin here with the crack growth rate corner which we shall denote as $\Delta K_{0}$ corresponding to an initial circular crack of radius, $\mathrm{a}_{0}$. In first approximation, we obtain [1]:

$$
N_{f}=\frac{\pi E^{2}}{2(\Delta \sigma)^{2}}
$$

It means that the propagation of the crack inside a fish eye is of the order of $10^{5}$ cycles. Therefore, the key parameter in gigacycle fatigue is initiation.

\section{Effect of loading condition}

The fish eye occurs in the high strength steel from a small inclusion of oxide, in plane strain conditions, with a plasticity located inside a plastic zone around an inclusion. In this case, the plane stress effect at the surface is not efficient facing the stress concentration due to a defect in steel where the UTS is close to 2000 $\mathrm{MPa}$. There is a competition between plane strain plasticity and plane stress plasticity.
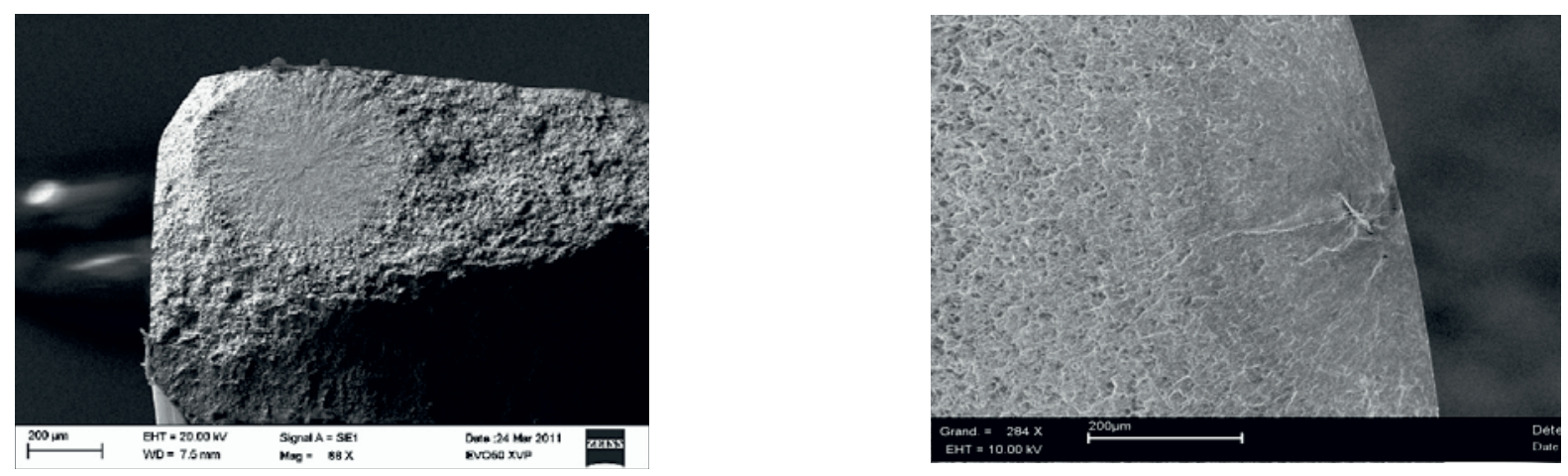

fig.2 Fish eye initiation in a flat specimen in tension- compression and in round specimen in torsion.

For high strength steel, the fatigue crack initiation location depends strongly of the number of cycles to failures. Roughly speaking, it is observed that the initiation is located at the surface of the specimen when the fatigue life does not exceed $10^{7}$ cycles according to the theory. But beyond this fatigue life, there is a competition between surface and internal initiation even in torsion loading This surprising competition is related to the high rate of inclusions in high strength steel. In torsion, the stress concentration around a MnS inclusion induces more plasticity than the shear stress at the surface. It is also interesting to notice the critical inclusion is not the same in torsion (sulphur) and in tension (oxide).

\section{Effect of microstructure transformation}

Through our own results and from the literature it is observed that the micro plasticity in the gigacycle fatigue induced more than dislocations sliding. Sometime, phase transformation, refining of the grain, twinning, and instability of the yield point, occur even when the loading is small during a very high number of cycles.

In high carbon content and in high strength steel, there is a transformation of the microstructure starting from the inclusion, in relation with the stress concentration and the stress field. It is difficult to understand this transformation which appears at the microscopic scale in a radius of 200 microns around an inclusion!

In high strength steels, the stability of the microstructure and the grain boundaries are involved in the plastic de formation to explain crack initiation in the fish e.ye It seems that martensite or bainite could be transformed inside butterfly wings, similar to what is observed in rolling contact fatigue. This mechanism is affected by the former grain boundaries [2].

\section{References}

1. C. Bathias ,PC. Paris, Gigacycle fatigue in mechanical practice ( Marcel Dekker, New York, 2005)

2. C. Bathias, Fatigue limit in metals (Wiley Focus, Hoboken, 2014) 